\title{
Could hematologic parameters be useful biomarkers for the diagnosis of endometriosis?
}

\author{
Turgut $\mathrm{A}^{1,2}$, Hocaoglu $\mathrm{M}^{2}$, Ozdamar $\mathrm{O}^{1,2}$, Usta $\mathrm{A}^{3}$, Gunay $\mathrm{T}^{2}$, Akdeniz $\mathrm{E}^{4}$ \\ Department of Obstetrics and Gynecology, Faculty of Medicine, Istanbul Medeniyet University, Istanbul, Turkey. \\ dr.meryemtaskiran@mail.com
}

\begin{abstract}
OBJECTIVES: The objective of this study was to evaluate the diagnostic value of the neutrophil-tolymphocyte ratio (NLR), lymphocyte-to-monocyte ratio (LMR), and mean platelet volume (MPV) in patients with endometriosis as compared with healthy controls.

BACKGROUND: Currently, no non-invasive diagnostic test of endometriosis has been implemented in clinical practice.

METHODS: A total of 121 women with endometriosis and 136 controls participated in this retrospective study. The extent of the disease in the patients with endometriosis was determined using the American Society of Reproductive Medicine revised classification. Sensitivities and specificities of NLR, LMR and MPV were evaluated by receiver-operating characteristic (ROC) analysis.

RESULTS: Patients with endometriosis had higher neutrophil counts, white blood cell (WBC) levels, NLR, MPV, and lower lymphocyte count and LMR than the control group. The cut-off values were found to be 1.6 for NLR at $87.6 \%$ sensitivity and $44.8 \%$ specificity and 8 for MPV at $75.2 \%$ sensitivity and $68.4 \%$ specficity. For LMR, the cut-off value was 5.6 with $66.1 \%$ sensitivity, $50 \%$ specificity. Patients with stages III or IV had significantly lower MPV $(p=0.039)$ and LMR levels $(p=0.016)$ than patients with stages I or II. CONCLUSION: NLR, LMR, and MPV may be used to distinguish patients with endometriosis from controls (Tab. 4, Fig. 4, Ref. 37). Text in PDF www.elis.sk.

KEY WORDS: CA-125, endometriosis, lymphocyte-to-monocyte ratio, mean platelet volume, neutrophil-to-lymphocyte ratio.
\end{abstract}

\section{Introduction}

Endometriosis is characterized by the presence of implants of abnormally placed tissue similar to endometrium, including glands and stroma, outside the uterine cavity $(1,2)$. It causes pelvic inflammation, which leads to pelvic pain, including dysmenorrhea, and infertility (3). The definitive diagnosis of endometriosis is made from histological evaluation of a lesion biopsied during surgery such as laparoscopy or laparotomy, which is an expensive and invasive procedure $(4,5)$. The diagnosis of endometriosis by means of a non-invasive diagnostic biomarker is a challenging problem (6). Although a variety of tests utilizing blood markers have been suggested as diagnostic measures for endometriosis, none have been implemented routinely in clinical practice (7).

Neutrophil-to-lymphocyte ratio (NLR) is affected by both innate immune response (mediated by neutrophils) and adaptive

${ }^{1}$ Department of Obstetrics and Gynecology, Faculty of Medicine, Istanbul Medeniyet University, Istanbul, Turkey, ${ }^{2}$ Department of Obstetrics and Gynecology, Goztepe Training and Research Hospital, Istanbul Medeniyet University, Istanbul, Turkey, ${ }^{3}$ Department of Obstetrics and Gynecology, Faculty of Medicine, Balikesir University, Balikesir, Turkey, and ${ }^{4}$ Division of Biostatistics, Faculty of Medicine, Marmara University, Istanbul Turkey

Address for correspondence: M. Hocaoglu, MD, Dr, Erkin cad. Goztepe Training and Research Hospital, Kadikoy, Istanbul, Turkey.

Phone: +90 5063718935, Fax: +216566 6614 immune response (mediated by lymphocytes) (8). NLR has been shown to be a reliable marker of systemic inflammation, which has been demonstrated in various studies $(9,10)$. The levels of circulating lymphocytes and monocytes are reflective of the immunological function in the peripheral blood (11). Lymphocyte to-monocyte ratio has been proposed as a surrogate marker for endothelial dysfunction and inflammation in distinct populations and it also has a prognostic and predictive value (12). The platelet activation has long been noticed in the pathophysiology of infection and inflammation. The mean platelet volume (MPV) is a reliable indicator of platelet size, which reflects platelet function and activation (13). It has been suggested that MPV has an important role as a marker of inflammation, disease activity, and efficacy of anti-inflammatory treatment in several chronic inflammatory disorders (14). However, the research in the area of NLR and MPV in the diagnosis of endometriosis is very limited and none of it investigates LMR in women with endometriosis $(15,16,17)$

Due to the presence of a chronic inflammatory state in patients with endometriosis, it was decided to conduct the current study while using NLR, LMR and MPV to investigate their roles in the diagnosis of endometriosis. The aim of this study was to evaluate the diagnostic value of NLR, LMR and MPV in Turkish patients with endometriosis as compared with a healthy control group. 

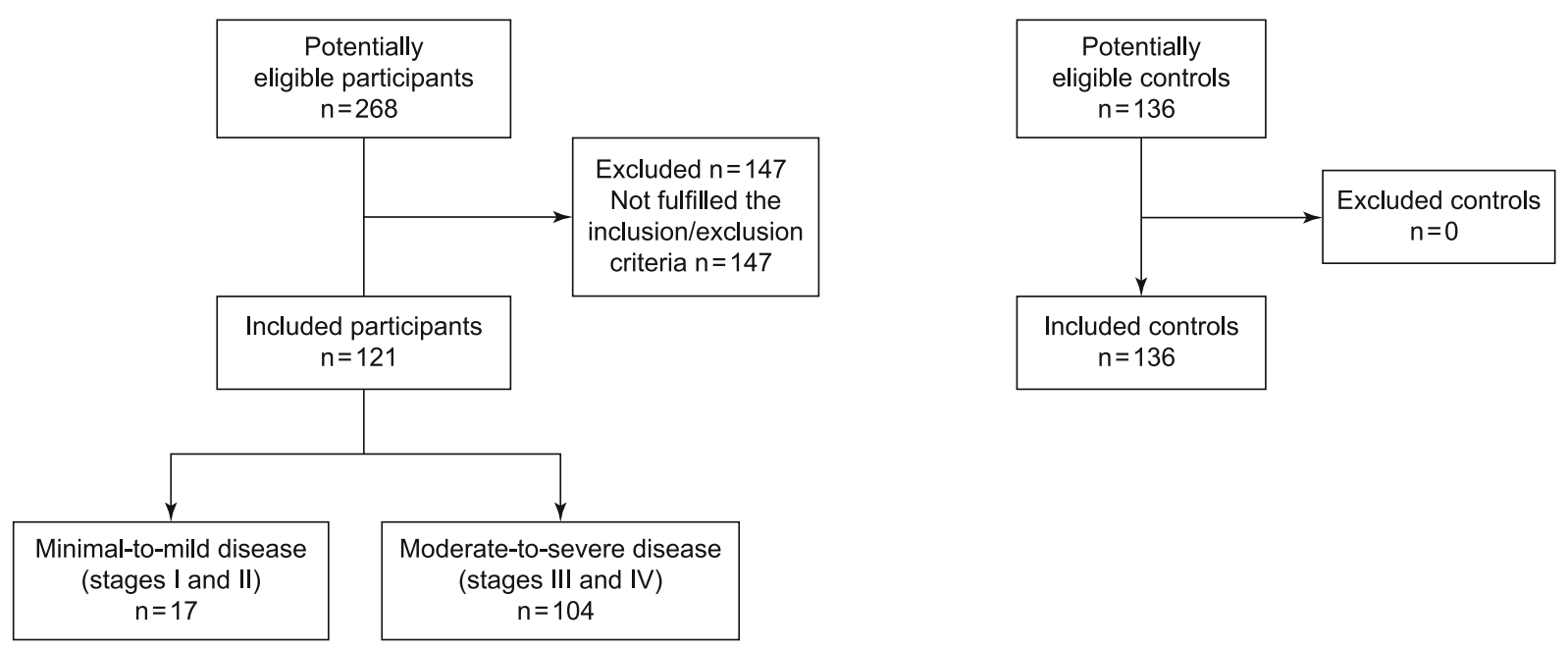

Fig. 1. STARD diagram for the flow of participants (Turkish patients with endometriosis and controls) throughout the study. (A) All 121 patients with endometriosis from 2012 to 2017 , who fulfilled the clinical diagnostic criteria were enrolled in this study. (B) Healthy controls (136 Turkish women) were also recruited.

\section{Materials and methods}

This retrospective, comparative, cohort study was carried out at the Department of Obstetrics and Gynecology of Istanbul Medeniyet University, Goztepe Training and Research Hospital, and was approved by the Institutional Review Board and Ethics Committee. The initial evaluation was made in 268 premenopausal women who underwent either laparoscopic or laparotomic surgery and had their diagnosis of endometriosis confirmed pathologically between January 2012 and February 2017 following surgery. A total of 121 women were recruited into the study according to the exclusion criteria. Patients were excluded if they had a confirmed pathology or clinical suspicion of endometrial pathology, leiomyoma or adenomyosis. The control group, consisted of 136 healthy women who presented at the gynecology clinic for a routine checkup and had no complaints or history of gynecological disease or cancer. Sample selection flow chart is given in Figure 1. Transvaginal ultrasonography (TVS) was performed on both groups during the gynecological examination. The exclusion criteria for the two groups included pregnancy, menopause, chronic diseases such as hematological, cardiac, kidney or liver diseases, prediabetes, diabetes mellitus, hypertension, hyperlipidemia asthma, connective tissue disorders, previous thrombosis, neoplastic disease, acutechronic inflammatory disorders, use of glucocorticoids, antineoplastic agents, anticoagulants, non-steroidal anti-inflammatory drugs, or oral contraceptives, hormonal treatment and smoking.

The clinical, demographic, laboratory, and surgical data were collected from hospital records and patient files. The preoperative complete blood count parameters (white blood cell, neutrophil, lymphocyte, monocyte and platelet counts, MPV, hemoglobin and hematocrit) and levels of CA- 125 were recorded. All patients were operated on during the follicular phase of the menstrual cycle, and all blood analyses were performed during the early follicular phase. The extent of the disease in patients with endometrio- sis was determined using the American Society of Reproductive Medicine (ASRM) revised classification while the severity of the disease was classified as minimal-to-mild disease (stages I-II) or moderate-to severe disease (stages III-IV) (1).

The blood samples were collected in EDTA tubes and processed using a hematology analyzer (Abbott CELL DYN 3700 , Boston, USA) for complete blood count analysis. CA-125 levels were measured using ARCHITECT i2000 (Abbott Diagnostics, Abbott Park, IL). The neutrophil-to-lymphocyte ratio (NLR) was calculated for both the patient and control groups by dividing the absolute neutrophil count by the absolute lymphocyte count. Lymphocyte-to-monocyte ratio (LMR) was calculated for both the patient and control groups by dividing the absolute lymphocyte count by the absolute monocyte count.

\section{Statistical analyses}

Statistical analyses were performed using R Statistical Software (www.r-project.org), a free software environment for statistical computing and graphics (18). The Shapiro-Wilk test, QQ and PP plots were used to analyze the data distribution. The continuous baseline characteristics of the groups were presented as median, interquartile range (IQR), minimum and maximum values depending on the data distribution while categorical values were defined as number (n) and percentage (\%). Parity, virginity, complaints and endometrioma variables were compared between stage I and II and stages III and IV in patient groups using Fisher's exact test. The Shapiro-Wilk test was used to analyze the data distribution. The Mann-Whitney U test was used for comparisons and the associated $\mathrm{p}$ values were given. The effect sizes of the tests were also given (19). The effect sizes for 2 by 2 chi-square test was given as the phi coefficient and Cramer's V for larger tables using "assocstats" function of the power analysis package in R (20, 21). The effect size for Mann-Whitney U test was calculated by dividing the $\mathrm{z}$ test statistic by the square root of the number of 
Tab. 1. Baseline characteristics of study group with endometriosis.

\begin{tabular}{lcc}
\hline & $\begin{array}{c}\text { No. of women } \\
(\mathrm{n}=121\end{array}$ & $\begin{array}{c}\text { Percentage } \\
(\%)\end{array}$ \\
\hline Age (years) Median (IQR) & $39(11)$ & \\
Min; max & $22-53$ & 36 \\
\hline Nulliparous & 43 & 64 \\
\hline Multiparous & 78 & 12 \\
\hline Virginity & 15 & 32 \\
\hline Chronic pelvic pain & 39 & 10 \\
\hline Primary infertility & 12 & 1 \\
\hline Secondary infertility & 2 & 8 \\
\hline Dysmenorrhea & 10 & 16 \\
\hline Abnormal uterine bleeding & 20 & 5 \\
\hline Dyspareunia & 6 & 6 \\
\hline Pelvic pain & 7 & 77 \\
\hline Endometrioma & 93 & 8 \\
I & 10 & 6 \\
II & 7 & 27 \\
III & 33 & 59 \\
IV & 71 & 14 \\
\hline Stages I-II (minimal-mild) & 17 & 86 \\
\hline Stages III-IV (moderate-severe) & 104 & \\
\hline Diameter of mass & $6(3)$ & \\
Median (IQR) & $2-16$ & \\
Min; max & & \\
\hline IQR, & & \\
\hline
\end{tabular}

IQR, interquartile range; Min, minimum; Max, maximum

cases $(21,22)$. Age-adjusted $p$ values were calculated using the logistic regression. The receiver-operating curve (ROC) analyses were constructed to evaluate diagnostic performances and optimal cut-off values for LMR, NLR and MPV in endometriosis patients. Youden's index (Maximum $=$ Sensitivity + Specificity -1$)$ was used as an optimization criterion for cut-off values (23). The area under the ROC curves was used to assess the discriminative the ability of LMR, NLR and MPV in endometriosis (24-26). Epi and verification programs were used for ROC curve estimations (27). The area under the ROC curve was calculated following the process outlined by Mason and Graham (25). The standard error of the area under the curve (AUC) was calculated based on the
Hanley and McNeil paper (26). The p-value produced for AUC was related to the Mann-Whitney U statistics. The AUCs of ROC curves were compared using the pROC program of $\mathrm{R}$ based on the method of Delong et al (28). For all analyses, a value of $\mathrm{p}<$ 0.05 was considered statistically significant.

\section{Results}

Overall, 121 patients with endometriosis were included in the study, of whom 17 had stage I or II and 104 had stage III or IV of endometriosis. The control group consisted of 136 healthy women who had presented at gynecology outpatient clinics for a routine check-up. The baseline characteristics of the endometriosis group are shown in Table 1. The median age of patients in the endometriosis group and healthy control group were 39 (interquartile range $(\mathrm{IQR})=11)$ years, and $38(\mathrm{IQR}=18)$ years, respectively. The patients with endometriosis were significantly older than the subjects in the control group (39 vs 35 years; $\mathrm{p}<$ $0.05)$. Thus, age was included as a covariate in the logistic regression model with the given variable to classify the control and endometriosis groups. After adjustment for age, the patients with endometriosis had significantly higher neutrophil counts, WBC levels, NLR, MPV, and lower lymphocyte count and LMR than the control group $(p<0.05)$. Mean platelet volume seemed to have the highest effect size among other variables (effect size $d$ $=0.401$ ). The comparisons of characteristics between the control group and patient group with endometriosis and age-adjusted $\mathrm{p}$ values are given in Table 2 . There were no statistically significant differences in respect of parity, virginity and complaints between patients with minimal-to-moderate disease (stages I and II) and moderate-to-severe disease (stages III and IV) $(\mathrm{p}>0.05)$. The percentage of endometrioma was determined to be much higher in the late stages of endometriosis $(p<0.001)$. The endometriosis group was also assessed according to the severity of the disease (Tab. 3). After adjustment for age, the patients with stage III or IV had significantly lower MPV counts $(\mathrm{p}=0.039$; effect size $\mathrm{d}$ $=0.142)$ and LMR $(p=0.016$; effect size $d=0.199)$ than patients with stage III or IV.

Tab. 2. Comparison of characteristics between the control group and patient group with endometriosis.

\begin{tabular}{|c|c|c|c|c|c|c|c|}
\hline \multirow{2}{*}{ Characteristics } & \multicolumn{2}{|c|}{ Control group $(n=136)$} & \multicolumn{2}{|c|}{ Patient group $(n=121)$} & \multirow[b]{2}{*}{$\mathrm{p}$} & \multirow{2}{*}{ p (age-adjusted) } & \multirow{2}{*}{ Effect size } \\
\hline & median (IQR) & $\min ; \max$ & median (IQR) & $\min ; \max$ & & & \\
\hline$\overline{\mathrm{CA}}-125, \mathrm{U} / \mathrm{mL}$ & - & - & $48(45.4)$ & $6.6 ; 643$ & - & - & - \\
\hline Age, years & $35(18)$ & $17 ; 51$ & $39(11)$ & $22 ; 53$ & $0.002 *$ & - & 0.189 \\
\hline WBC & $6.65(1.9)$ & $3 ; 11.2$ & $7(2.6)$ & $3.9 ; 71$ & $0.008 *$ & $0.0024 *$ & 0.165 \\
\hline Neutrophil & $3.55(1.53)$ & $1.5 ; 6.8$ & $4.4(1.9)$ & $2 ; 17.2$ & $<0.001 *$ & $<0.001 *$ & 0.286 \\
\hline Lymphocyte, $\times 10^{3} / \mu 1$ & $2.15(0.8)$ & $1.1 ; 3.9$ & $2(0.8)$ & $0.4 ; 3.8$ & $<0.001 *$ & $0.002 *$ & 0.153 \\
\hline Monocyte, $\times 10^{3} / \mu 1$ & $0.4(0.2)$ & $0.1 ; 0.9$ & $0.4(0.2)$ & $0.2 ; 0.7$ & 0.636 & 0.3967 & 0.030 \\
\hline Hematocrit, \% & $37.45(3.73)$ & $25 ; 43.9$ & $35.8(5.2)$ & $23.6 ; 312$ & $0.003 *$ & 0.354 & 0.185 \\
\hline Hemoglobin & $12.4(1.2)$ & $7.6 ; 14.7$ & $11.6(2)$ & $7.4 ; 83$ & $<0.001 *$ & 0.883 & 0.251 \\
\hline NLR & $1.70(0.8)$ & $0.74 ; 4.4$ & $2.18(0.86)$ & $0.8 ; 31.75$ & $<0.001 *$ & $0.00032 *$ & 0.350 \\
\hline LMR & $5.6(3.7)$ & $2.4 ; 22$ & $4.8(2.64)$ & $0.5 ; 11.5$ & $0.005^{*}$ & $<0.001^{*}$ & 0.175 \\
\hline MPV & $7.7(1.33)$ & $5.8-11.7$ & $9.1(2)$ & $5.3-13.5$ & $<0.001 *$ & $<0.001 *$ & 0.401 \\
\hline
\end{tabular}

IQR, Interquartile range; WBC, white blood cell; NLR, neutrophil-to-lymphocyte ratio; LMR, lymphocyte-to-monocyte ratio; MPV, mean platelet volume. * p < 0.05 was considered statistically significant. IQR: Interquartile range 
Tab. 3. Comparison of laboratory characteristics between patients with minimal-to-moderate disease (stages I or II) and moderate-to-severe disease (stages III or IV).

\begin{tabular}{|c|c|c|c|c|c|c|c|}
\hline \multirow{2}{*}{ Variables } & \multicolumn{2}{|c|}{ Stages I-II $(n=17)$} & \multicolumn{2}{|c|}{ Stages III-IV $(n=104)$} & \multirow{2}{*}{$\mathrm{p}$} & \multirow{2}{*}{ Age_adjusted $\mathrm{p}$} & \multirow{2}{*}{ Effect size } \\
\hline & Median (IQR) & Min-Max & Median (IQR) & Min-Max & & & \\
\hline Age & $46(6)$ & $26-53$ & $38.5(10)$ & $22-51$ & $<0.05^{*}$ & - & 0.216 \\
\hline WBC & $7.1(0.8)$ & $4.6-14.7$ & $7(2.73)$ & $3.9-71$ & 0.506 & 0.692 & 0.041 \\
\hline Neutrophil & $4.2(1.1)$ & $2.4-9.9$ & $4.55(2.05)$ & $2-17.2$ & 0.402 & 0.638 & 0.066 \\
\hline Lymphocyte & $2.2(0.8)$ & $1.4-3.8$ & $2(0.8)$ & $0.4-3.4$ & 0.281 & 0.316 & 0.066 \\
\hline Monocyte & $0.3(0.1)$ & $0.2-0.6$ & $0.4(0.3)$ & $0.2-0.7$ & $0.015^{*}$ & 0.131 & 0.160 \\
\hline NLR & $1.9(1.1)$ & $1-3.43$ & $2.2(0.84)$ & $0.8-31.75$ & 0.318 & 0.336 & 0.067 \\
\hline MPV & $10.1(1.8)$ & $7.2-11$ & $9(1.93)$ & $5.3-13.5$ & 0.055 & $0.039 *$ & 0.142 \\
\hline CA-125 IU/mL & $25(23.3)$ & $8-72$ & $51.5(46)$ & $6.6-643$ & $0.048^{*}$ & 0.132 & 0.157 \\
\hline PLT & $282(100)$ & $188-327$ & $263(87.75)$ & $140-503$ & 0.498 & 0.603 & 0.021 \\
\hline HCT & $37(2)$ & $28.4-41.7$ & $35.4(5.23)$ & $23.6-312$ & 0.687 & 0.766 & 0.071 \\
\hline $\mathrm{HB}$ & $11.8(1.1)$ & 8.8-14 & $11.6(2.2)$ & $7.4-12.22$ & 0.854 & 0.839 & 0.062 \\
\hline LMR & $6(2.7)$ & $3.16-11$ & $4.63(2.25)$ & $0.5-11.5$ & $<0.001 *$ & $0.016^{*}$ & 0.199 \\
\hline
\end{tabular}

p, p value significant at 0.05 level; IQR, interquartile range; min, minimum; max, maximum; CA-125, serum cancer antigen; WBC, white blood cell; NLR, neutrophil-tolymphocyte ratio; MPV, mean platelet volume; LMR, lymphocyte-to-monocyte ratio; HCT, hematocrit; HB, hemoglobin, * p < 0.05 was considered statistically significant. IQR: Interquartile range, Mann-Whitney test. Values are expressed as medians.

Tab. 4. Sensitivity, specificity, positive predictive value and negative predictive value of blood count parameters LMR, NLR and MPV for detection of endometriosis.

\begin{tabular}{lccccccccc}
\hline Biomarkers & $\begin{array}{c}\text { AUC } \\
(95 \% \mathrm{Cl})\end{array}$ & $\begin{array}{c}\text { Sensitivity } \\
(\%)\end{array}$ & $\begin{array}{c}\text { Specificity } \\
(\%)\end{array}$ & PPV & NPV & LR- & LR+ & Cut-off value & $\mathrm{p}$ \\
\hline $\mathrm{WBCs} / \mathrm{mL}$ & $\begin{array}{c}0.596 \\
(0.526-0.665)\end{array}$ & 59.5 & 55.9 & 55.5 & 61.8 & 1.35 & 0.72 & 6.8 & 0.004 \\
\hline Neutrophils $/ \mathrm{mL}$ & $\begin{array}{c}0.666 \\
(0.5998-0.7322)\end{array}$ & 76 & 51.4 & 58.2 & 70.7 & 1.57 & 0.47 & 3.6 & $<0.001$ \\
\hline NLR & $\begin{array}{c}0.702 \\
(0.6398-0.7662)\end{array}$ & 87.6 & 44.8 & 58.6 & 80.3 & 1.59 & 0.28 & 1.60 & $<0.001$ \\
\hline MPV & $\begin{array}{c}0.732 \\
(0.666-0.798)\end{array}$ & 75.2 & 68.4 & 67.9 & 75.6 & 2.38 & 0.36 & 8 & $<0.001$ \\
\hline LMR & $\begin{array}{c}0.601 \\
(0.532-0.670)\end{array}$ & 66.1 & 50 & 37.6 & 45.9 & 0.68 & 1.32 & 5.6 & 0.002 \\
\hline
\end{tabular}

WBC, white blood cell; NLR, neutrophil-to-lymphocyte ratio; LMR, lymphocyte-to-monocyte ratio; MPV, mean platelet volume; AUC, area under the curve; CI, confidence interval; PPV, positive predictive value; NPV, negative predictive value; LR: likelihood ratio, ${ }^{*} \mathrm{p}<0.05$ was considered statistically significant

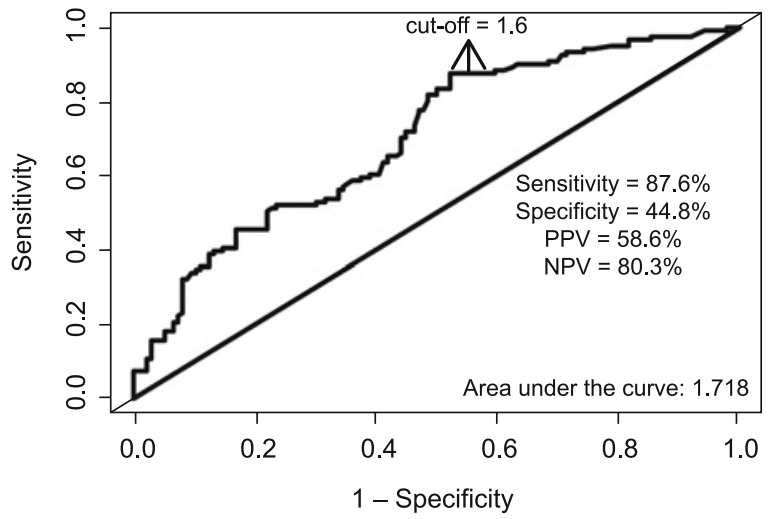

Fig. 2. Receiver-operating characteristic (ROC) curve of neutrophilto-lymphocyte ratio (NLR) for differentiating endometriosis from healthy control.

There was a significant difference between the patient group and control group in respect of biomarkers, WBC, neutrophil, LMR, NLR, and MPV. Thus, these biomarkers were further investigated for potential cut-off points and area under curve (AUC). The sensitivity, specificity, positive predictive value and nega-

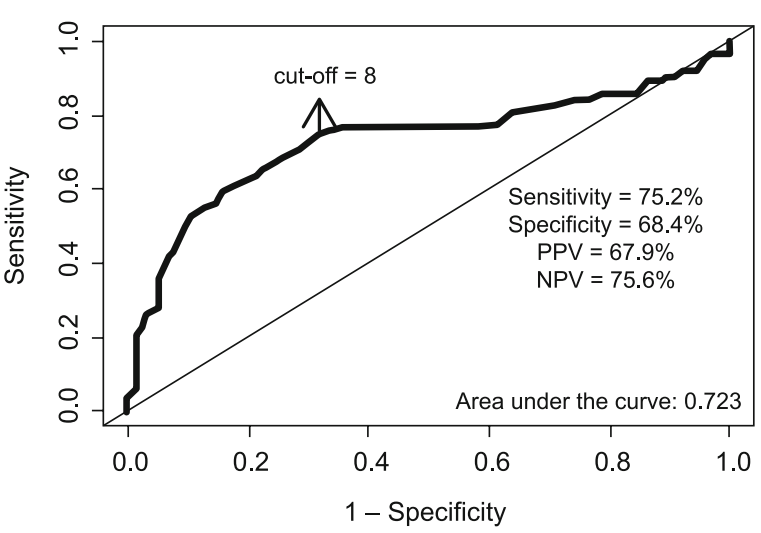

Fig. 3. Receiver-operating characteristic (ROC) curve of mean platelet volume (MPV) for differentiating endometriosis from healthy controls

tive predictive value for these biomarkers are shown in Table 4. ROC analysis indicated that the combined marker had a higher AUC (0.766) than other biomarkers. The ROC analyses indicate that the $\mathrm{p}$ values associated with the given biomarkers were all $<$ 0.05 . Thus, all AUCs were significantly different from 0.5 , which 


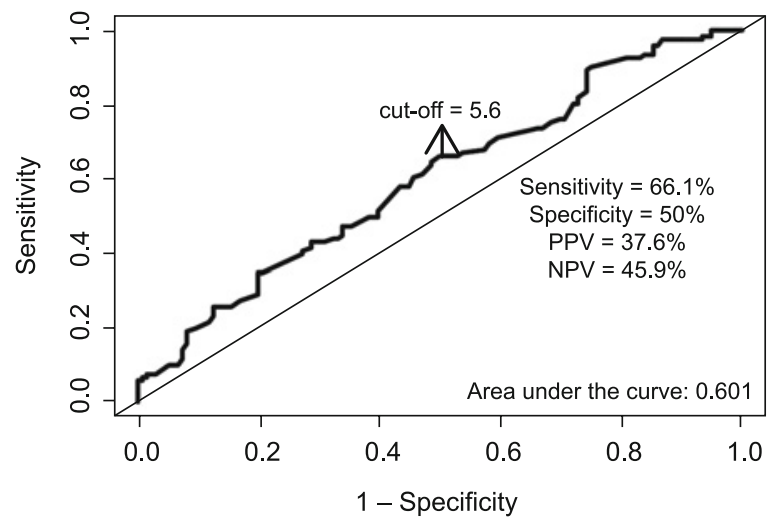

Fig. 4. Receiver-operating characteristic (ROC) curve of lymphocyte to monocyte ratio (LMR) for differentiating endometriosis from healthy controls

shows that these biomarkers are all good indicators for anticipating endometriosis. The AUC for NLR was 0.702 with $95 \%$ CI from 0.639 to 0.766 (Fig. 2). The best cut-off for NLR was 1.6. At this cut-off point, the sensitivity was $87.6 \%$, specificity 44.8 $\%$, positive predictive value $58.6 \%$, and negative predictive value $80.3 \%$. For MPV, the AUC was 0.732 (95\% CI 0.666-0.798), and the cutoff value was 8 with $75.2 \%$ sensitivity, $68.4 \%$ specificity, positive predictive value of $67.9 \%$, and negative predictive value of $75.6 \%$ (Fig. 3). For LMR, the AUC was 0.601 (95\% CI $0.532-0.670$ ) and the cutoff value was 5.6 with $66.1 \%$ sensitivity, $50 \%$ specificity, positive predictive value of $37.6 \%$, and negative predictive value of $45.9 \%$ (Fig. 4 ).

\section{Discussion}

Cancer antigen 125 (CA-125) has been widely used for the diagnosis and follow-up of patients with endometriosis. The sensitivity and specificity of CA-125 with a cutoff level of $35 \mathrm{IU} / \mathrm{mL}$ in this study were $55.8 \%$ and $92.8 \%$, respectively, which was similar to the results of meta-analyses conducted in 23 studies involving 2,866 patients. It is often elevated in advanced endometriosis while sensitivity is especially low in early-stage disease. The sensitivity and specificity of the serum CA-125 measurement in the diagnosis of endometriosis grade III/IV have been reported as $47 \%$ and $89 \%$, respectively (29). CA-125 is expressed in some derivatives of coelomic epithelium. It has been found to be elevated in the serum of patients with epithelial ovarian carcinomas, endometrium and fallopian tube carcinomas, and gastrointestinal and breast malignancies (30). Also, the timing of blood collection for CA-125 in relation to the menstrual cycle is known to significantly affect this test (31)

Endometriosis is an inflammatory condition. It is known that endometrial cells that reflux into the peritoneal cavity secrete chemokines creating a feed-forward loop that stimulates the infiltration of immune cells (32). Subclinical peritoneal inflammation in patients with endometriosis is demonstrated by increased white blood cells, especially macrophages, and higher cytokine levels in the peritoneal fluid (17). Increased neutrophil and decreased lymphocyte counts resulted in an increase in NLR in patients with endometriosis when compared with the healthy control group. There have been few studies to date assessing the role of NLR as a diagnostic marker in patients with endometriosis as compared with the healthy control group. In a retrospective study using NLR to differentiate between endometriosis cases and control subjects (benign tumor group and healthy control group), Cho et al. reported that NLR with a cut-off value of 2.01 had sensitivity and specificity of $59.7 \%$ and $60.1 \%$, respectively, which is consistent with the current findings (15). It was also determined that NLR combined with CA-125 had the sensitivity of $69.3 \%$ and specificity of $83.9 \%$ when 55.7 was set as the cut-off value (15). In a prospective study of patients with endometriosis and patients with benign ovarian cysts performed by Sayan et al, the combined marker (NLR and CA-125) had the highest sensitivity at $80.0 \%$ when compared to NLR, CA-125, and interleukin 8 alone, with specificity of $86.0 \%$, which was lower than the specificity of CA125 , the marker with the highest specificity (16). In a retrospective comparative study of women with endometriosis and benign ovarian cysts, Tokmak et al suggested that the best NLR cut-off value was 1.9 with $70 \%$ sensitivity and $74 \%$ specificity (17). In addition, the cut-off value for the combined marker of NLR and CA-125 was determined to be 41.0 with $80 \%$ sensitivity and 82 $\%$ specificity (17). The results reported by Sayan et al and Tokmak et al are inadequate for differentiating the patients with endometriosis from healthy control subjects $(16,17)$.

Mean platelet volume (MPV) is the size of platelets, and an increase in this value is an indicator of large more reactive platelets resulting from increased platelet turnover. In a study by Guo et al, it was reported that the platelet count, WBC count, MPV and platelet activation rate in the peritoneal fluid from women with endometriosis were significantly elevated when compared with those of women without endometriosis (33). In the current study, higher MPV levels were determined in patients with endometriosis in comparison with healthy women. Mean platelet volume with a cut-off value of 8 has the sensitivity and specificity of $75.2 \%$ and $68.4 \%$, respectively. Similarly, Tokmak et al found that MPV levels in patients with endometrioma were higher than those in the control group with benign ovarian cysts (17). However, sensitivity, specificity, positive predictive value, and negative predictive value of MPV are not analyzed in the study by Tokmak et al (17).

The lymphocyte-to-monocyte ratio (LMR) has been studied as an inflammatory marker in local or systemic inflammation, previous history of infection, and inflammatory diseases (12). In current literature, lower LMR values were assessed in inflammatory diseases (34). To the best of our knowledge, this is the first report describing the predictive role of LMR in the diagnosis of endometriosis. The results from this study indicate that lower LMR levels were determined in patients with endometriosis in comparison with healthy women. The lymphocyte-to-monocyte ratio with a cut-off value of 5.6 has the sensitivity and specificity of $66.1 \%$ and $50 \%$, respectively. It turned out that LMR was less sensitive than NLR and MPV. 
The endometriosis group was also evaluated according to the severity of the disease. Our study findings showed no differences in NLR between patients at stage I or II and those at stage III or IV. Also, the results of the current study were inconsistent with previous reports that NLR is significantly different between minimal-mild and moderate-severe diseases $(15,17)$. The discrepancy between the previous and present findings may be attributed to relatively few patients with stage I or II in our study cohort. Likewise, Kim et al (35) and Yavuzcan et al (36) reported that the severity of endometriosis was not related to the NLR value. In our study, MPV and LMR were found to be statistically different in the stages of endometriosis. Conversely, Yavuzcan et al stated that there was no significant relationship between MPV and advanced-stage endometriosis (36). Interestingly, a comparison of MPV based on disease severity showed differences, which was significantly decreased in patients with stage III or IV of the disease. These findings may seem to be incompatible with the pathophysiology of endometriosis. But also, the discrepancy between the change in MPV levels and severity may be attributed to the fact that both low and high levels of MPV indicate inflammation. It seems that the size of circulating platelets is dependent on the intensity of systemic inflammation with contrasting features of MPV in high- and low-grade inflammatory diseases and course of anti-inflammatory treatment. Disease-specific and cardiovascular confounding factors affect the direction of MPV changes (14). Therefore, while low-grade inflammatory conditions are associated with high levels of MPV, the high-grade inflammatory diseases such as active rheumatoid arthritis or attacks of familial Mediterranean fever are associated with low levels of MPV (37).

The current study has some limitations. The sample size was moderate, thus further research involving a larger population size is needed to confirm the clinical utility. As it was a retrospective analysis, the data entry errors could be a possible source of bias. Data such as body mass index are missing, which could also contribute to the confounding effects. It was not possible to assess CA- 125 levels in the healthy control group.

\section{Conclusion}

The results of this study have shown that NLR and MPV in patients with endometriosis are significantly higher and LMR is lower than those in healthy controls without endometriosis. Lymphocyte-to-monocyte (LMR) ratio and MPV may be considered as useful biomarkers for both the diagnosis of endometriosis and prediction of the disease severity. These biomarkers are practical, easy and inexpensive tests. Nevertheless, they warrant further evaluation in prospective, controlled studies with larger sample sizes and additional markers.

\section{References}

1. Hocaoglu M, Turgut A, Ozdamar O, Aslan A, Demirer S, Usta A, Ekdeniz E, Karateke A. Abdominal wall endometriosis in patients with a history of cesarian section. Ann Ital Chir 2018; 89: 425-430.
2. Dordevic M, Jovanovic B, Mitrovic S, Dordevic G, Radovanovic D, Sazdanovic P. Abdominal rectus muscle endometriosis after Cesarean section. Extrapelvic localization of endometriosis. Bratisl Lek Listy 2010; 111 (6): 345-348.

3. Pitonak J, Galova J, Bernasovska J. Association of two selected polymorphisms with developed endometriosis in women from Slovakia. Bratisl Lek Listy 2016; 117 (8): 452-455.

4. American Society for Reproductive Medicine. Revised American Society for Reproductive Medicine classification of endometriosis Fertil Steril 1996; 67: 817.

5. Duffy JM, Arambage K, Correa FJ et al. Laparoscopic surgery for endometriosis. Cochrane Database Syst Rev 2014; 3: CD011031.

6. Casper RF. Introduction: A focus on the medical management of endometriosis. Fertil Steril 2017; 107: 521-522.

7. Nisenblat V, Bossuyt PM, Shaikh R et al. Blood biomarkers for the non-invasive diagnosis of endometriosis. Cochrane Database Syst Rev 2016; 05: CD012179.

8. Korkmaz M, Korkmaz H, Küçüker F, Ayyildiz SN, Çankaya S. Evaluation of the association of sleep apnea-related systemic inflammation with CRP, ESR, and neutrophil-to-lymphocyte ratio. Med Sci Monit $2015 ; 13 ; 21: 477-481$.

9. Gezer C, Ekin A, Solmaz U, Sahingoz Yildirim AG, Dogan A, Ozeren M. Identification of preterm birth in women with threatened preterm labour between 34 and 37 weeks of gestation. J Obstet Gynaecol 2018; 9: 1-6.

10. Verit FF, Cetin O, Yildirim O, Keskin S, Yucel O, Yalcinkaya S. Neutrophil to lymphocyte ratio is superior to platelet to lymphocyte ratio as an early predictor of moderate/severe ovarian hyperstimulation syndrome. J Obstet Gynaecol 2014; 34: 639-643.

11. Ji H, Niu X, Yin L et al. Ratio of Immune Response to Tumor Burden Predicts Survival Via Regulating Functions of Lymphocytes and Monocytes in Diffuse Large B-Cell Lymphoma. Cell Physiol Biochem 2018; 45: 951-961.

12. Balta S., Demirer Z., Aparci M., Yildirim A.O., Ozturk C. The lymphocyte-monocyte ratio in clinical practice. J Clin Pathol 2016; 69: 88.

13. Ekin A, Gezer C, Kulhan G, Avci ME, Taner CE. Can platelet count and mean platelet volume during the first trimester of pregnancy predict preterm premature rupture of membranes? J Obstet Gynaecol Res 2015; 41: 23-28.

14. Gasparyan AY, Ayvazyan L, Mikhailidis DP, Kitas GD. Mean platelet volume: a link between thrombosis and inflammation? Curr Pharm Des 2011; 17: 47-58.

15. Cho S, Cho H, Nam A et al. Neutrophil-to-lymphocyte ratio as an adjunct to CA-125 for the diagnosis of endometriosis. Fertil Steril 2008; 90: 2073-2709.

16. Sayan CD, Ozaksit MG, Sarikaya E, Eryilmaz OG, Mollamahmutoglu L, Deveer R. Serum interleukin-8, CA-125 levels, neutrophilto-lymphocyte ratios, and combined markers in the diagnosis of endometriosis. Turk J Med Sci 2013; 43: 417-423.

17. Tokmak A, Yildirim G, Öztaş E et al. Use of Neutrophil-to-Lymphocyte Ratio Combined With CA-125 to Distinguish Endometriomas From Other Benign Ovarian Cysts. Reprod Sci 2016; 23: 795-802.

18. $\mathbf{R}$ Development Core Team. R: A language and environment for statistical computing. R Foundation for Statistical Computing, Vienna, Austria 2008. ISBN 3-900051-07-0, URL http://www.R-project.org. 
912-918

19. Sullivan GM, Feinn R. Using effect size - or why the $P$ value is not enough. J Grand Med Educ 2012; 4: 279-282.

20. Gravetter FJ, Wallnau LB. Statistics for the behavioral sciences. Cengage Learning 2016.

21. Pallant J. SPSS survival manual 2016; 6th Edition. McGraw-Hill Education (UK).

22. Cohen J. Statistical power analysis for the behavioral sciences. 1988.

23. Youden WJ. Index for rating diagnostic tests. Cancer 1950; 3: 32.

24. Xavier R, Robin X, Turck $\mathbf{N}$ et al. pROC: an open-source package for $\mathrm{R}$ and $\mathrm{S}+$ to analyze and compare ROC curves. BMC Bioinformatics 2011; 17: 12 .

25. Mason SJ, Graham NE. Areas beneath the relative operating characteristics (ROC) and relative operating levels (ROL) curves: Statistical significance and interpretation. Q J R Meteorol Soc 2002; 128: 2145.

26. Hanley JA, McNeil BJ. The meaning and use of the area under a receiver-operating characteristic (ROC) curve. Radiology 1982; 143: 29.

27. Carstensen B, Plummer M, Laara E, Hills M. Epi: A Package for Statistical Analysis in Epidemiology 2016; R package version 2.7. URL https://CRAN.R-project.org/package=Epi.

28. DeLong ER, DeLong DM, Clarke-Pearson DL. Comparing the areas under two or more correlated receiver operating characteristic curves: a nonparametric approach. Biometrics 1988; 44: 837-845.

29. Mol BW, Bayram N, Lijmer JG et al. The performance of CA-125 measurement in the detection of endometriosis: a meta-analysis. Fertil Steril 1988; 70: 1101.

30. Chen FP, Soong YK, Lee N, Lo SK. The use of serum CA-125 as a marker for endometriosis in patients with dysmenorrhea for monitoring therapy and for recurrence of endometriosis. Acta Obstet Gynecol Scand 1998; 77: 665-670.
31. Spaczynski RZ, Duleba AJ. Diagnosis of endometriosis. Semin Reprod Med 2003; 193-208.

32. McKinnon BD, Kocbek V, Nirgianakis K, Bersinger NA, Mueller MD. Kinase signaling pathways in endometriosis: potential targets for non-hormonal therapeutics. Hum Reprod Update 2016; 4: 22.

33. Guo SW, Du Y, Liu X. Platelet-derived TGF-b1 mediates the downmodulation of NKG2D expression and may be responsible for impaired natural killer (NK) cytotoxicity in women with endometriosis. Hum Reprod 2016; 31: 1462-1474.

34. Demirdal T, Sen P. The significance of neutrophil-lymphocyte ratio, platelet-lymphocyte ratio and lymphocyte-monocyte ratio in predicting peripheral arterial disease, peripheral neuropathy, osteomyelitis and amputation in diabetic foot infection. Diabetes Res Clin Pract 2018; 144 : $118-1125$.

35. Kim SK, Park JY, Jee BC, Suh CS, Kim SH. Association of the neutrophil-to-lymphocyte ratio and CA 125 with the endometriosis score. Clin Exp Repro Med 2014; 41: 151-157.

36. Yavuzcan A, Cağlar M, Üstün Y et al. Evaluation of mean platelet volume, neutrophil/ lymphocyte ratio and platelet/lymphocyte ratio in advanced stage endometriosis with endometrioma. J Turk German Gynecol Ass 2013; 210-215.

37. Balta I, Balta S, Koryurek OM et al. Mean platelet volume is associated with aortic arterial stiffness in patients with Behçet's disease without significant cardiovascular involvement. J Eur Acad Dermatol and Venereol 2014; $28:$ 1388-1393.

Received April 9, 2019. Accepted July 2, 2019. 